\title{
The dual eigenvalue problems of the conformable fractional Sturm-Liouville problems
}

\section{Yan-Hsiou Cheng ${ }^{1 *}$ (i)}

\section{"Correspondence:}

yhcheng@tea.ntue.edu.tw

'Department of Mathematics and

Information Education, National

Taipei University of Education, 106

Taipei, Taiwan, R.O.C.

\section{Springer}

\begin{abstract}
In this paper, we are concerned with the eigenvalue gap and eigenvalue ratio of the Dirichlet conformable fractional Sturm-Liouville problems. We show that this kind of differential equation satisfies the Sturm-Liouville property by the Prüfer substitution. That is, the $n$th eigenfunction has $n-1$ zero in $(0, \pi)$ for $n \in \mathbb{N}$. Then, using the homotopy argument, we find the minimum of the first eigenvalue gap under the class of single-well potential functions and the first eigenvalue ratio under the class of single-barrier density functions. The result of the eigenvalue gap is different from the classical Sturm-Liouville problem.
\end{abstract}

MSC: Primary 34A08; secondary 34B24; 26A33

Keywords: Conformable fractional derivatives; Sturm-Liouville problem; Eigenvalue gap; Eigenvalue ratio

\section{Introduction}

Consider the Sturm-Liouville problem with conformable fractional derivatives of order $\alpha, 0<\alpha \leq 1$,

$$
\begin{aligned}
& -D_{x}^{\alpha} D_{x}^{\alpha} y+q(x) y=\lambda \rho(x) y, \quad 0<x<\pi, \\
& y(0)=y(\pi)=0 .
\end{aligned}
$$

Here, $\lambda$ is the spectral parameter, $\rho(x), q(x) \in L_{\alpha}^{2}(0, \pi)$ are real, $\rho(x)>0$ a.e., and $D_{x}^{\alpha}$ is the conformable fractional derivative of order $\alpha, 0<\alpha \leq 1$. This type of fractional derivative was first studied by Khalil et al. in 2014 [17]. One can also see [1, 6] for some basic properties and main results. The conformable fractional Sturm-Liouville problems (CFSLP) have been studied in various areas of science and in many fields in engineering, see $[4,11,13]$ and the references therein. In particular, Zhao and Luo [21] gave physical and geometrical interpretations of the conformable derivative and generalized the definition of conformable fractional derivative by means of linear extended Gâteaux derivative to general conformable fractional derivative.

C) The Author(s) 2021. This article is licensed under a Creative Commons Attribution 4.0 International License, which permits use, sharing, adaptation, distribution and reproduction in any medium or format, as long as you give appropriate credit to the original author(s) and the source, provide a link to the Creative Commons licence, and indicate if changes were made. The images or other third party material in this article are included in the article's Creative Commons licence, unless indicated otherwise in a credit line to the material. If material is not included in the article's Creative Commons licence and your intended use is not permitted by statutory regulation or exceeds the permitted use, you will need to obtain permission directly from the copyright holder. To view a copy of this licence, visit http://creativecommons.org/licenses/by/4.0/. 
In 2017, Al-Refai and Abdeljawad [3] showed that the eigenvalues of the CFSLP with separated boundary conditions which include conformable fractional derivatives of order $\alpha, 1 / 2<\alpha \leq 1$, are all real and simple. In particular, the eigenfunctions corresponding to distinct eigenvalues are $\alpha$-orthogonal with respect to the weight function $\rho(x)$. They also gave the fractional Rayleigh quotient to identify the eigenvalues. Recently, Mortazaasl and Akbarfam [19] investigated the trace formula of the eigenvalues and studied the inverse nodal problem of the CFSLP with conformable fractional boundary conditions of order $\alpha, 0<\alpha \leq 1$. They showed that the eigenvalues of the CFSLP with separated boundary conditions are all real and simple, and the $n$th eigenfunction has precisely $n-1$ nodes in the interval $(0, \pi)$ for sufficiently large $n$. They also solved the uniqueness problem of the inverse nodal problem and gave a reconstruction formula by using the nodal data. Meanwhile, Adalar and Ozkan [2] studied the inverse spectral problems of the CFSLP and gave uniqueness theorems according to the Weyl function, two eigenvalues-sets, and the sequences which consist of eigenvalues and norming constants.

For the classical Sturm-Liouville problem $-y^{\prime \prime}+q(x) y=\lambda \rho(x) y$, the optimal estimates of eigenvalue gaps and eigenvalue ratios have attracted much attention for a long time, see $[5,10,14-16,18]$. What we call the dual problem here is the focus on the issue related to the eigenvalue gap of the Schrödinger equation $-y^{\prime \prime}+q(x) y=\lambda y$ and the eigenvalue ratio of the string equation $-y^{\prime \prime}=\lambda \rho(x) y$. It was showed that, for the Dirichlet Schrödinger equation, the constant potential function gives the minimal eigenvalue gaps $\lambda_{2}-\lambda_{1}$ when the potential function $q$ is assumed to be convex [18], symmetric single-well [5], or single-well [14]. On the restriction of the space $E[h, H, M] \equiv\left\{q: h \leq q \leq H, \int_{0}^{\pi} q(x) d x=M\right\}$, the symmetric 1-step function gives the minimal eigenvalue gap [10]. On the other hand, for the Dirichlet string equation, the constant density function gives the minimal eigenvalue ratio $\lambda_{2} / \lambda_{1}$ when the density function $\rho$ is assumed to be concave, symmetric single-barrier [15], or single-barrier [14]. On the restriction of the space $E[h, H, M]$, the symmetric 1-step function gives the minimal eigenvalue ratio [16]. It shall be mentioned that Cheng et al. [9] in 2014 investigated the dual eigenvalue problems of the Dirichlet Sturm-Liouville problem with the $p$-Laplacian operator and showed that $\lambda_{2}-\lambda_{1} \geq 2^{p}-1$ for the Schrödinger type equation with $\rho(x) \equiv 1$ and single-well potential functions, while $\lambda_{2} / \lambda_{1} \geq 2^{p}$ for the string type equation with $q(x) \equiv 0$ and single-barrier density functions. In particular, the equalities hold when the potential function and the density function are constant in each case respectively.

In the present paper, we consider the first eigenvalue gap $\lambda_{2}-\lambda_{1}$ and eigenvalue ratio $\lambda_{2} / \lambda_{1}$ of the Dirichlet CFSLP. By the help of the Prüfer substitution, we show the $n$th eigenfunction has $n-1$ zeros in $(0, \pi)$ for $n \in \mathbb{N}$. This generalizes one result of [19]. Then, using the homotopy argument, we find the minimum of $\lambda_{2}-\lambda_{1}$ and $\lambda_{2} / \lambda_{1}$.

Theorem 1.1 Consider the CFSLP with $\rho(x) \equiv 1$ and single-well potential functions with a transition point at $\pi / 2$. Then

$$
\lambda_{2}-\lambda_{1} \geq \frac{3 \alpha^{2} \pi^{2-2 \alpha}}{2^{2-2 \alpha}}
$$


Theorem 1.2 Consider the CFSLP with $q(x) \equiv 0$ and single-barrier density functions with a transition point at $\pi / 2$. Then

$$
\frac{\lambda_{2}}{\lambda_{1}} \geq 4
$$

Equality holds if and only if $\rho$ is constant.

Remark 1 By setting $\rho(x) \equiv 1$ and $q(x) \equiv 0$ for all $x \in[0, \pi]$, the first two eigenvalues are

$$
\sqrt{\lambda_{1}}=\alpha \pi^{1-\alpha}, \quad \sqrt{\lambda_{2}}=2 \alpha \pi^{1-\alpha},
$$

and the corresponding normalized eigenfunctions are

$$
y_{1}(x, 0)=\sqrt{\frac{2 \alpha}{\pi^{\alpha}}} \sin \left(\pi^{1-\alpha} x^{\alpha}\right), \quad y_{2}(x, 0)=\sqrt{\frac{2 \alpha}{\pi^{\alpha}}} \sin \left(2 \pi^{1-\alpha} x^{\alpha}\right) .
$$

We find that the eigenvalue gap is

$$
\lambda_{2}-\lambda_{1}=3 \alpha^{2} \pi^{2-2 \alpha},
$$

while the eigenvalue ratio is

$$
\frac{\lambda_{2}}{\lambda_{1}}=4
$$

This shows that the minimum of $\lambda_{2}-\lambda_{1}$ does not occur at the constant potential. This is not the same as the classical Sturm-Liouville problems. The constant potential is the minimizer of $\lambda_{2}-\lambda_{1}$ only if $\alpha=1$. However, the minimizer of the eigenvalue ratio is the same as the classical Sturm-Liouville problems.

This paper is organized as follows. In Sect. 2, we give some basic definitions and properties of the conformable fractional calculus. We also give some lemmas that would help us to prove the main theorems. Finally, we prove our main results, Theorems 1.1-1.2, in Sect. 3.

\section{Preliminaries}

In this section, we first introduce basic definitions and properties of the conformable fractional calculus theory that can be found in $[1,17]$.

Definition 2.1 Let $f:[0, \infty) \rightarrow \mathbb{R}$ be a given function and $0<\alpha \leq 1$.

(a) The conformation fractional derivative of $f$ of order $\alpha$ is defined by

$$
D_{x}^{\alpha} f(x) \equiv \lim _{h \rightarrow 0} \frac{f\left(x+h x^{1-\alpha}\right)-f(x)}{h}, \quad D_{x}^{\alpha} f(0)=\lim _{x \rightarrow 0^{+}} D_{x}^{\alpha} f(x)
$$

for all $x>0$. 
(b) The conformation fractional integral of $f$ of order $\alpha$ is defined by

$$
I_{\alpha} f(x) \equiv \int_{0}^{x} f(t) \mathrm{d}_{\alpha} t=\int_{0}^{x} t^{\alpha-1} f(t) d t
$$

for all $x>0$. Here, the last integral is the usual Riemann improper integral.

Theorem 2.1 Let $f$, $g$ be $\alpha$-differentiable in $x \in(0, \infty)$.

(a) $D_{x}^{\alpha}(a f+b g)=a D_{x}^{\alpha} f+b D_{x}^{\alpha} g$ for all $a, b \in \mathbb{R}$.

(b) $D_{x}^{\alpha}\left(x^{p}\right)=\alpha x^{p-\alpha}$ for $p \in \mathbb{R}$.

(c) $D_{x}^{\alpha}(c)=0$ for $c \in \mathbb{R}$.

(d) $D_{x}^{\alpha}(f g)=g D_{x}^{\alpha} f+f D_{x}^{\alpha} g$.

(e) $D_{x}^{\alpha}(f / g)=\frac{g D_{x}^{\alpha} f-f D_{x}^{\alpha} g}{g^{2}}$.

(f) Iff is differentiable, then $D_{x}^{\alpha} f(x)=x^{1-\alpha} f^{\prime}(x)$.

Next, we introduce two lemmas that will be used when we prove the main theorems. In the proof of the main theorems, we transfer the problems of finding the eigenvalue gap/ratio of the CFSLP to the problems of finding the zero gap/ratio of some trigonometric equations. There is a $p$-Laplacian version in [9], and we set $p=2$ in this paper.

Lemma 2.2 Define $f(s)=\sqrt{s} \cot \left(\frac{\pi}{2} \sqrt{s}\right)$ and let $s_{1}, s_{2}$ be the first two zeros of $f(s)=-f(s-m)$ for $m>0$, respectively. Then

$$
t_{2}-t_{1} \geq 3
$$

Lemma 2.3 Define $g(s)=\frac{1}{s} \tan (s)$ and let $s_{1}, s_{2}$ be the first two zeros of $g(s)=-g(s m)$ for $m>1$, respectively. Then

$$
\frac{s_{2}}{s_{1}} \geq 2
$$

At the end of this section, we show that the CFSLP satisfies the Sturm-Liouville property, i.e., the CFSLP has infinitely many eigenvalues $\left\{\lambda_{n}\right\}_{n \in \mathbb{N}}$ satisfying

$$
\lambda_{1}<\lambda_{2}<\cdots<\lambda_{n}<\cdots,
$$

and the corresponding eigenfunction $y\left(x, \lambda_{n}\right)$ has $n-1$ zeros in $(0, \pi)$. Define the modified Prüfer substitution

$$
y(x)=r(x) \sin (\theta(x)), \quad D_{x}^{\alpha} y(x)=r(x) \cos (\theta(x)) .
$$

Then

$$
\frac{D_{x}^{\alpha} y(x)}{y(x)}=\frac{\cos (\theta(x))}{\sin (\theta(x))}
$$

We find

$$
D_{x}^{\alpha}\left(\frac{D_{x}^{\alpha} y(x)}{y(x)}\right)=\frac{y(x) D_{x}^{\alpha} D_{x}^{\alpha} y(x)-D_{x}^{\alpha} y(x) D_{x}^{\alpha} y(x)}{y^{2}(x)}=\frac{(q(x)-\lambda) y^{2}(x)-\left(D_{x}^{\alpha} y(x)\right)^{2}}{y^{2}(x)}
$$


and

$$
D_{x}^{\alpha}\left(\frac{\cos (\theta(x))}{\sin (\theta(x))}\right)=\frac{-x^{1-\alpha} \theta^{\prime}(x) \sin ^{2}(\theta(x))-x^{1-\alpha} \theta^{\prime}(x) \cos ^{2}(\theta(x))}{\sin ^{2}(\theta(x))}=-\frac{x^{1-\alpha} \theta^{\prime}(x)}{\sin ^{2}(\theta(x))}
$$

This implies that

$$
\begin{aligned}
\theta^{\prime}(x) & =-x^{\alpha-1} \sin ^{2}(\theta(x)) \frac{(q(x)-\lambda \rho(x)) y^{2}(x)-\left(D_{x}^{\alpha} y(x)\right)^{2}}{y^{2}(x)} \\
& =x^{\alpha-1}\left[\cos ^{2}(\theta(x))+(\lambda \rho(x)-q(x)) \sin ^{2}(\theta(x))\right] .
\end{aligned}
$$

It can be showed that $F(x, t) \equiv x^{\alpha-1}\left[\cos ^{2}(t)+(\lambda \rho(x)-q(x)) \sin ^{2}(t)\right]$ is Lipschitz in $t$. In particular, by analyzing the function $\theta^{\prime}$, we can prove Lemma 2.4. The proof is similar to [20, Lemma 2.3] so we omit it here. One can also refer to $[7,8,12]$.

Lemma 2.4 Consider the CFSLP. We have the following results:

(a) The function $\theta(\cdot, \lambda)$ satisfying $\theta(0, \lambda)=0$ is continuous and strictly increasing in $\lambda$;

(b) If $\theta\left(x_{n}, \lambda\right)=n \pi$ for $n \in \mathbb{N}$, then $\theta(x, \lambda)>n \pi$ for all $x>x_{n}$;

(c) For any $a \in(0,1]$,

$$
\lim _{\lambda \rightarrow \infty} \theta(a, \lambda)=\infty, \quad \lim _{\lambda \rightarrow-\infty} \theta(a, \lambda)=0 .
$$

By Lemma 2.4, there exists a sequence of eigenvalues $\left\{\lambda_{n}\right\}_{n=1}^{\infty}$ satisfying

$$
\lambda_{1}<\lambda_{2}<\cdots<\lambda_{n}<\cdots \rightarrow \infty
$$

and the corresponding eigenfunction $y_{n}$ has $n-1$ zeros in $(0, \pi), n \in \mathbb{N}$. In the rest of the paper, we assume that $\left\{\lambda_{n}, y_{n}(x)\right\}_{n \in \mathbb{N}}$ are normalized eigenpairs, i.e., $\int_{0}^{\pi} \rho(t)\left|y_{n}(t)\right|^{2} \mathrm{~d}_{\alpha} t=1$. It can be showed that the first two eigenfunctions $y_{1}$ and $y_{2}$ intersect at most twice.

Lemma 2.5 The equation $\left|y_{1}(x)\right|=\left|y_{2}(x)\right|$ has at most two solutions in $(0, \pi)$.

Proof Let $x_{0}$ be the zero of $y_{2}(x)$ in $(0, \pi)$, and let $\phi_{1}(x)=y_{2}(x) / y_{1}(x)$. Then, for $x \in\left(0, x_{0}\right)$, we have

$$
D_{x}^{\alpha}\left(\frac{y_{2}(x)}{y_{1}(x)}\right)=\frac{y_{1}(x) D_{x}^{\alpha} y_{2}(x)-y_{2}(x) D_{x}^{\alpha} y_{1}(x)}{y_{1}^{2}(x)}
$$

Let $\phi(x)=y_{1}(x) D_{x}^{\alpha} y_{2}(x)-y_{2}(x) D_{x}^{\alpha} y_{1}(x)$. Then

$$
\begin{aligned}
D_{x}^{\alpha} \phi(x) & =y_{1}(x) D_{x}^{\alpha}\left(D_{x}^{\alpha} y_{2}(x)\right)-y_{2}(x) D_{x}^{\alpha}\left(D_{x}^{\alpha} y_{1}(x)\right) \\
& =\left(\lambda_{1}-\lambda_{2}\right) \rho(x) y_{1}(x) y_{2}(x) \\
& <0
\end{aligned}
$$

for $x \in\left(0, x_{0}\right)$. Since $\phi(0)=0$ and $D_{x}^{\alpha} \phi(x)=x^{1-\alpha} \phi^{\prime}(x)$, we find that $\phi(x)$ is strictly decreasing, and hence $\phi(x)<0$ on $\left(0, x_{0}\right)$. Furthermore, we obtain $\frac{y_{2}(x)}{y_{1}(x)}$ is strictly decreasing on 
$\left(0, x_{0}\right)$. This implies that $y_{1}(x)$ and $y_{2}(x)$ have at most one intersection point in $\left(0, x_{0}\right)$. Similarly, $y_{1}(x)$ and $-y_{2}(x)$ have at most one intersection point in $\left(x_{0}, \pi\right)$. The proof is complete.

Lemma 2.6 Let $q(x, s)$ and $\rho(t, s)$ be one-parameter families of piecewise continuous functions such that $\frac{\partial}{\partial s} q(x, s)$ and $\frac{\partial}{\partial s} \rho(x, s)$ exist. Denote by $\left\{\lambda_{n}(s), y_{n}(x, s)\right\}_{n \in \mathbb{N}}$ the normalized eigenpairs. Then we have

$$
\frac{d}{d s} \lambda_{n}(s)=\int_{0}^{\pi}\left|y_{n}(t, s)\right|^{2} \frac{\partial}{\partial s} q(t, s) d_{\alpha} t-\lambda_{n}(s) \int_{0}^{\pi}\left|y_{n}(t, s)\right|^{2} \frac{\partial}{\partial s} \rho(t, s) d_{\alpha} t .
$$

Proof Consider the CFSLP with one-parameter family of piecewise functions $q(x, s)$ and $\rho(x, s)$ :

$$
\begin{aligned}
& -D_{x}^{\alpha} D_{x}^{\alpha} y_{n}(x, s)+q(x, s) y_{n}(x, s)=\lambda_{n}(s) \rho(x, s) y_{n}(x, s), \quad 0<x<\pi, \\
& y_{n}(0, s)=y_{n}(\pi, s)=0 .
\end{aligned}
$$

Denote $\dot{f}=\frac{\partial}{\partial s} f$. We applied the variation analysis on the homotopy of the CFSLP. One can refer to [16]. Differentiating the above equations with respect to $s$, we can obtain the variation equation

$$
\begin{aligned}
- & D_{x}^{\alpha} D_{x}^{\alpha} \dot{y}_{n}(x, s)+\dot{q}(x, s) y_{n}(x, s)+q(x, s) \dot{y}_{n}(x, s) \\
& =\dot{\lambda}_{n}(s) \rho(x, s) y_{n}(x, s)+\lambda_{n}(s) \dot{\rho}(x, s) y_{n}(x, s)+\lambda_{n}(s) \rho(x, s) \dot{y}_{n}(x, s), \quad 0<x<\pi,
\end{aligned}
$$

and

$$
\dot{y}_{n}(0, s)=\dot{y}_{n}(\pi, s)=0 .
$$

Then we have

$$
\begin{aligned}
& \dot{\lambda}_{n}(s) \rho(x, s) y_{n}^{2}(x, s) \\
& \quad=\dot{y}_{n}(x, s) D_{x}^{\alpha} D_{x}^{\alpha} y_{n}(x, s)-y_{n}(x, s) D_{x}^{\alpha} D_{x}^{\alpha} \dot{y}_{n}(x, s)+\dot{q}(x, s) y_{n}^{2}(x, s)-\lambda_{n}(s) \dot{\rho}(x, s) y_{n}^{2}(x, s) .
\end{aligned}
$$

Hence, by the $\alpha$-integration by parts,

$$
\begin{aligned}
\dot{\lambda}_{n}(s)= & \int_{0}^{\pi} \dot{\lambda}_{n}(s) \rho(x, s) y_{n}^{2}(x, s) d_{\alpha} t \\
= & \int_{0}^{\pi}\left(\dot{y}_{n}(x, s) D_{x}^{\alpha} D_{x}^{\alpha} y_{n}(x, s)-y_{n}(x, s) D_{x}^{\alpha} D_{x}^{\alpha} \dot{y}_{n}(x, s)\right) d_{\alpha} t \\
& +\int_{0}^{\pi} \dot{q}(x, s) y_{n}^{2}(x, s) d_{\alpha} t-\int_{0}^{\pi} \lambda_{n}(s) \dot{\rho}(x, s) y_{n}^{2}(x, s) d_{\alpha} t \\
= & {\left[\dot{y}_{n}(x, s) D_{x}^{\alpha} y_{n}(x, s)-y_{n}(x, s) D_{x}^{\alpha} \dot{y}_{n}(x, s)\right]_{0}^{\pi} } \\
& +\int_{0}^{\pi}\left(D_{x}^{\alpha} \dot{y}_{n}(x, s) D_{x}^{\alpha} y_{n}(x, s)-D_{x}^{\alpha} y_{n}(x, s) D_{x}^{\alpha} \dot{y}_{n}(x, s)\right) d_{\alpha} t \\
& +\int_{0}^{\pi} \dot{q}(x, s) y_{n}^{2}(x, s) d_{\alpha} t-\lambda_{n}(s) \int_{0}^{\pi} \dot{\rho}(x, s) y_{n}^{2}(x, s) d_{\alpha} t
\end{aligned}
$$




$$
=\int_{0}^{\pi} \dot{q}(x, s) y_{n}^{2}(x, s) d_{\alpha} t-\lambda_{n}(s) \int_{0}^{\pi} \dot{\rho}(x, s) y_{n}^{2}(x, s) d_{\alpha} t .
$$

This completes the proof.

Following from Lemma 2.6, we have, for $\rho(x) \equiv 1$,

$$
\frac{d}{d s}\left(\lambda_{2}(s)-\lambda_{1}(s)\right)=\int_{0}^{\pi}\left(\left|y_{2}(t, s)\right|^{2}-\left|y_{1}(t, s)\right|^{2}\right) \frac{\partial}{\partial s} q(t, s) d_{\alpha} t
$$

and, for $q(x) \equiv 0$,

$$
\frac{d}{d s}\left(\frac{\lambda_{2}(s)}{\lambda_{1}(s)}\right)=\frac{\lambda_{2}(s)}{\lambda_{1}(s)} \int_{0}^{\pi}\left(\left|y_{2}(t, s)\right|^{2}-\left|y_{1}(t, s)\right|^{2}\right) \frac{\partial}{\partial s} \rho(t, s) d_{\alpha} t .
$$

\section{Proof of the main theorems}

Proof of Theorem 1.1 For $M>0$, we denote

$$
Q_{M} \equiv\{0 \leq q(x) \leq M: q \text { is single-well with a transition point at } \pi / 2\}
$$

Then $E[q] \equiv\left(\lambda_{2}-\lambda_{1}\right)[q]$ is bounded on $Q_{M}$, and hence $E[q]$ attains its minimum at some $q_{0}$ in $Q_{M}$. Taking $q(x) \in Q_{M}$ that will be assigned in the following, we define by $q(x, s) \equiv s q(x)+$ $(1-s) q_{0}(x)$ a one-parameter family of potential functions where $s \in[0,1]$. By Lemma 2.5, there exist $0 \leq x_{-}<x_{0}<x_{+} \leq \pi$ such that $y_{2}\left(x_{0}, 0\right)=0$ and

$$
\left|y_{2}(x, 0)\right|^{2}-\left|y_{1}(x, 0)\right|^{2} \begin{cases}>0 & \text { on }\left(0, x_{-}\right) \cup\left(x_{+}, \pi\right), \\ <0 & \text { on }\left(x_{-}, x_{+}\right) .\end{cases}
$$

In the following, we show that the case $x_{-} \leq \pi / 2 \leq x_{+}$is the only possibility.

(a) Assume $x_{-} \leq \pi / 2 \leq x_{+}$. Let

$$
q(x)= \begin{cases}q_{0}\left(x_{-}\right) & \text {on }\left(0, \frac{\pi}{2}\right) \\ q_{0}\left(x_{+}\right) & \text {on }\left(\frac{\pi}{2}, \pi\right)\end{cases}
$$

By the optimality of $q_{0}$, we find that

$$
0 \leq\left.\frac{d}{d s}\left(\lambda_{2}(s)-\lambda_{1}(s)\right)\right|_{s=0}=\int_{0}^{\pi}\left(q(t)-q_{0}(t)\right)\left(\left|y_{2}(t, s)\right|^{2}-\left|y_{1}(t, s)\right|^{2}\right) d_{\alpha} t<0
$$

This implies that $q_{0}(x)=q(x)$ on $(0, \pi)$ is a one-step function.

(b) Assume $x_{-}>\pi / 2$ (the case for $x_{+}<\pi / 2$ is similar). Without loss of generality, we assume that $q$ is of the form

$$
q(x)= \begin{cases}0 & \text { on }\left(0, x_{-}\right) \\ M & \text { on }\left(x_{-}, \pi\right)\end{cases}
$$

Since $y_{n}(x, 0)$ is normalized, we find

$$
\int_{0}^{x_{-}}\left(\left|y_{2}(t, 0)\right|^{2}-\left|y_{1}(t, 0)\right|^{2}\right) d_{\alpha} t>0
$$


and

$$
\int_{x_{-}}^{\pi}\left(\left|y_{2}(t, 0)\right|^{2}-\left|y_{1}(t, 0)\right|^{2}\right) d_{\alpha} t<0
$$

By the optimality of $q_{0}$, we find that

$$
\begin{aligned}
0 \leq & \left.\frac{d}{d s}\left(\lambda_{2}(s)-\lambda_{1}(s)\right)\right|_{s=0} \\
= & \int_{0}^{\pi}\left(q(t)-q_{0}(t)\right)\left(\left|y_{2}(t, 0)\right|^{2}-\left|y_{1}(t, 0)\right|^{2}\right) d_{\alpha} t \\
\leq & -q_{0}\left(\frac{\pi}{2}\right) \int_{0}^{x_{-}}\left(\left|y_{2}(t, 0)\right|^{2}-\left|y_{1}(t, 0)\right|^{2}\right) d_{\alpha} t \\
& +\left(M-q_{0}\left(x_{+}\right)\right) \int_{x_{-}}^{\pi}\left(\left|y_{2}(t, 0)\right|^{2}-\left|y_{1}(t, 0)\right|^{2}\right) d_{\alpha} t \\
\leq & 0 .
\end{aligned}
$$

Hence, the only possibility is $q_{0}(x)=q(x)$ on $(0, \pi)$. Furthermore, in this case, the second eigenfunction can be expressed by

$$
y_{2}(x)= \begin{cases}c \sin \left(\frac{\sqrt{\lambda_{2}}}{\alpha} x^{\alpha}\right) & \text { on }\left(0, x_{-}\right), \\ d \sin \left(\frac{\sqrt{\lambda_{2}-M}}{\alpha}(\pi-x)^{\alpha}\right) & \text { on }\left(x_{-}, \pi\right) .\end{cases}
$$

Since $\pi / 2<x_{-}<x_{0}$, we find $\frac{\sqrt{\lambda_{2}}}{\alpha}(\pi / 2)^{\alpha}<\pi<\frac{\sqrt{\lambda_{2}-M}}{\alpha}(\pi / 2)^{\alpha}$, and then $\sqrt{\lambda_{2}}<\sqrt{\lambda_{2}-M}$. But this is impossible and hence this case is refused.

By the above discussion, we may assume

$$
q_{0}(x)= \begin{cases}0 & \text { on }\left(0, \frac{\pi}{2}\right) \\ m & \text { on }\left(\frac{\pi}{2}, \pi\right)\end{cases}
$$

for some $m>0$. In this case, the eigenfunction corresponding to the eigenvalue $\lambda$ can be expressed by

$$
y_{2}(x)= \begin{cases}c \sin \left(\frac{\sqrt{\lambda}}{\alpha} x^{\alpha}\right) & \text { on }\left(0, \frac{\pi}{2}\right), \\ d \sin \left(\frac{\sqrt{\lambda-m}}{\alpha}(\pi-x)^{\alpha}\right) & \text { on }\left(\frac{\pi}{2}, \pi\right) .\end{cases}
$$

In particular, $\lambda$ is an eigenvalue if $\lambda$ is a solution of

$$
\left.\frac{D_{x}^{\alpha} \sin \left(\frac{\sqrt{\lambda}}{\alpha} x^{\alpha}\right)}{\sin \left(\frac{\sqrt{\lambda}}{\alpha} x^{\alpha}\right)}\right|_{x=\frac{\pi}{2}}=\left.\frac{D_{x}^{\alpha} \sin \left(\frac{\sqrt{\lambda-m}}{\alpha}(\pi-x)^{\alpha}\right)}{\sin \left(\frac{\sqrt{\lambda-m}}{\alpha}(\pi-x)^{\alpha}\right)}\right|_{x=\frac{\pi}{2}}
$$

or, equivalently, letting $\hat{\alpha}=\left(\frac{\pi}{2}\right)^{\alpha} / \alpha$,

$$
\sqrt{\lambda} \cot (\hat{\alpha} \sqrt{\lambda})=-\sqrt{\lambda-m} \cot (\hat{\alpha} \sqrt{\lambda-m}) .
$$


Here, $\hat{\alpha} \in[\pi / 2, \infty)$ is decreasing in $\alpha \in(0,1]$. In particular, let $\phi(\lambda)=\sqrt{\lambda} \cot (\hat{\alpha} \sqrt{\lambda}), f(t)=$ $\sqrt{t} \cot (\pi \sqrt{t} / 2), \lambda=(\pi /(2 \hat{\alpha}))^{2} t$, and $m=(\pi /(2 \hat{\alpha}))^{2} \theta$. Then $\phi(\lambda)=-\phi(\lambda-m)$ is equivalent to $f(t)=-f(t-\theta)$. By Lemma 2.2 , we find $t_{2}-t_{1} \geq 3$ or, equivalently,

$$
\lambda_{2}-\lambda_{1} \geq \frac{3 \pi^{2}}{4 \hat{\alpha}^{2}}=\frac{3 \alpha^{2} \pi^{2-2 \alpha}}{2^{2-2 \alpha}} .
$$

The proof is complete.

Proof of Theorem 1.2 For $M>1$, define

$$
\Omega_{M}=\left\{\frac{1}{M} \leq \rho(x) \leq M: \rho \text { is single-barrier with a transition point at } \pi / 2\right\} .
$$

Then $R[\rho] \equiv\left(\frac{\lambda_{2}}{\lambda_{1}}\right)[\rho]$ is bounded on $\Omega_{M}$, and hence $R[\rho]$ attains its minimum at some $\rho_{0}$ in $\Omega_{M}$. Now, for $\rho(x) \in \Omega_{M}$, define $\rho(x, s)=s \rho(x)+(1-s) \rho_{0}(x)$ to be a one-parameter family of density functions, where $s \in[0,1]$. Using the similar argument as the proof of Theorem 1.1, one can show that the optimal $\rho_{0}$ must be of the form

$$
\rho_{0}(x)=\left\{\begin{array}{ll}
1 & \text { on }\left(0, \frac{\pi}{2}\right), \\
L & \text { on }\left(\frac{\pi}{2}, \pi\right),
\end{array} \quad \text { or } \quad \rho_{0}(x)= \begin{cases}L & \text { on }\left(0, \frac{\pi}{2}\right) \\
1 & \text { on }\left(\frac{\pi}{2}, \pi\right)\end{cases}\right.
$$

for some $L \geq 1$. Without loss of generality, we assume that the first case holds. In this case, the eigenfunction corresponding to the eigenvalue $\lambda$ can be expressed as

$$
y(x)=\left\{\begin{array}{l}
c \sin \left(\frac{\sqrt{\lambda}}{\alpha} x^{\alpha}\right) \quad \text { on }\left(0, \frac{\pi}{2}\right) \\
d \sin \left(\frac{\sqrt{\lambda L}}{\alpha}(\pi-x)^{\alpha}\right) \quad \text { on }\left(\frac{\pi}{2}, \pi\right) .
\end{array}\right.
$$

In particular, $\lambda$ is an eigenvalue if $\lambda$ is a solution of

$$
\left.\frac{D_{x}^{\alpha} \sin \left(\frac{\sqrt{\lambda}}{\alpha} x^{\alpha}\right)}{\sin \left(\frac{\sqrt{\lambda}}{\alpha} x^{\alpha}\right)}\right|_{x=\frac{\pi}{2}}=\left.\frac{D_{x}^{\alpha} \sin \left(\frac{\sqrt{\lambda L}}{\alpha}(\pi-x)^{\alpha}\right)}{\sin \left(\frac{\sqrt{\lambda L}}{\alpha}(\pi-x)^{\alpha}\right)}\right|_{x=\frac{\pi}{2}}
$$

or, equivalently, for $\hat{\alpha}=\left(\frac{\pi}{2}\right)^{\alpha} / \alpha$,

$$
\sqrt{L} \tan (\hat{\alpha} \sqrt{\lambda})=-\tan (\hat{\alpha} \sqrt{\lambda L})
$$

Let $m=\sqrt{L}$ and $\lambda=s^{2} / \hat{\alpha}^{2}$. Then we obtain $m \tan s=-\tan (s m)$. By Lemma 2.3, we find $\frac{s_{2}}{s_{1}} \geq 4$ or equivalently

$$
\frac{\lambda_{2}}{\lambda_{1}}=\frac{s_{2}}{s_{1}} \geq 4
$$

In particular, for $\rho(x) \equiv 1$ and $q(x) \equiv 0$ for all $x \in[0, \pi]$, the first two eigenvalues are

$$
\sqrt{\lambda_{1}}=\alpha \pi^{1-\alpha}, \quad \sqrt{\lambda_{2}}=2 \alpha \pi^{1-\alpha},
$$


and we find

$$
\frac{\lambda_{2}}{\lambda_{1}}=4
$$

This shows that the minimum of $\lambda_{2} / \lambda_{1}$ occurs at the constant density.

\section{Acknowledgements}

The author's work is partially supported by the Ministry of Science and Technology of the Republic of China under the grant number MOST 109-2115-M-152-001.

\section{Funding}

Not applicable.

\section{Availability of data and materials}

Not applicable.

\section{Competing interests}

The author declares that they have no competing interests.

\section{Authors' contributions}

The author read and approved the final manuscript.

\section{Publisher's Note}

Springer Nature remains neutral with regard to jurisdictional claims in published maps and institutional affiliations.

Received: 15 May 2021 Accepted: 30 August 2021 Published online: 30 September 2021

\section{References}

1. Abdeljawad, T.: On conformable fractional calculus. J. Comput. Appl. Math. 279, 57-66 (2015)

2. Adalar, I., Sinan Ozkan, A.: Inverse problems for a conformable fractional Sturm-Liouville operator. J. Inverse III-Posed Probl. 28(6), 775-782 (2020). https://doi.org/10.1515/jiip-2019-0058

3. Al-Refai, M., Abdeljawad, T.: Fundamental results of conformable Sturm-Liouville eigenvalue problems. Complexity 2017, Article ID 3720471 (2017). https://doi.org/10.1155/2017/3720471

4. Anderson, D.R., Ulness, D.J.: Properties of the Katugampola fractional derivative with potential application in quantum mechanics. J. Math. Phys. 56(6), 063502 (2015)

5. Ashbaugh, M.S., Benguria, R.: Optimal lower bound for the gap between the first two eigenvalues of one-dimensional Schrödinger operators with symmetric single-well potentials. Proc. Am. Math. Soc. 105, 419-424 (1989)

6. Atangana, A., Baleanu, D.. Alsaedi, A.: New properties of conformable derivative. Open Math. 13, 889-898 (2015)

7. Binding, P.A., Drábek, P.: Sturm-Liouville theory for the $p$-Laplacian. Studia Sci. Math. Hung. 40, 373-396 (2003)

8. Birkhoff, G., Rota, G.C.: Ordinary Differential Equations. Wiley, New York (1989)

9. Cheng, Y.H., Lian, W.C., Wang, W.C.: The dual eigenvalue problems for p-Laplacian. Acta Math. Hung. 142(1), 132-151 (2014)

10. Chern, H.H., Shen, C.L.: On the maximum and minimum of some functionals for the eigenvalue problem of Sturm-Liouville type. J. Differ. Equ. 107, 68-79 (1994)

11. Chung, W.S.: Fractional Newton mechanics with conformable fractional derivative. J. Comput. Appl. Math. 290 150-158 (2015)

12. Coddington, E.A., Levinson, N.: Theory of Ordinary Differential Equations. McGraw-Hill, New York (1955)

13. Eslami, M., Rezazadeh, H.: The first integral method for Wu-Zhang system with conformable time fractional derivative. Calcolo 53(3), 475-485 (2016)

14. Horvath, M.: On the first two eigenvalues of Sturm-Liouville operators. Proc. Am. Math. Soc. 131, 1215-1224 (2002)

15. Huang, M.J.: On the eigenvalue ratio for vibrating strings. Proc. Am. Math. Soc. 127, 1805-1813 (1999)

16. Keller, J.B.: The minimum ratio of two eigenvalues. SIAM J. Appl. Math. 31, 485-491 (1976)

17. Khalila, R., Al Horania, M., Yousefa, A., Sababheh, M.: A new definition of fractional derivative. J. Comput. Appl. Math. $264,65-70(2014)$

18. Lavine, R.: The eigenvalue gap for one-dimensional convex potentials. Proc. Am. Math. Soc. 121, $815-821$ (1994)

19. Mortazaasl, $H_{\text {., }}$ Jodayree Akbarfam, A.: Trace formula and inverse nodal problem for a conformable fractional Sturm-Liouville problem. Inverse Probl. Sci. Eng. 28(4), 524-555 (2020)

20. Wang, W.C., Cheng, Y.H., Lian, W.C.: Inverse nodal problems for the $p$-Laplacian with eigenparameter dependent boundary conditions. Math. Comput. Model. 54, 2718-2724 (2011)

21. Zhao, D., Luo, H.: General conformable fractional derivative and its physical interpretation. Calcolo 54, $903-917$ (2017) 